\title{
Percutaneous Transapical approach in the Treatment of Paravalvular Leak
}

\author{
Weiqiang Ruan \\ Sichuan University West China Hospital \\ Qin Yang \\ Sichuan University West China Hospital \\ Changping Gan \\ Sichuan University West China Hospital \\ Ke Lin ( $\square$ pangzai356249@126.com ) \\ Sichuan University West China Hospital https://orcid.org/0000-0002-2406-2260
}

\section{Research article}

Keywords: paravalvular leak, transapical closure, residual regurgitation

Posted Date: October 29th, 2020

DOI: https://doi.org/10.21203/rs.3.rs-97669/v1

License: (c) (i) This work is licensed under a Creative Commons Attribution 4.0 International License. Read Full License 


\section{Abstract}

Background: Paravalvular leaks (PVLs) occur in up to $17 \%$ of patients after valve replacement. Due to the high morbidity and mortality associated with re-operation, percutaneous transapical (TA) closure has been introduced as an alternative to the surgical repair and percutaneous transcatheter closure in the treatment of PVLs. Here, we evaluated the outcomes of patients who underwent TA-closure for PVLs at the our centre.

Methods: This study analyzed records of 12 patients with 13 problematic mechanical valves who underwent treatment at our hospital from April 2015 to November 2018. All procedures were performed under general anesthesia, with preoperative CT reconstruction and guidance by three-dimensional transesophageal echocardiography (3D-TEE).

Results: Our data showed that all the TA punctures were successful with delivery sheath sizes that ranged from 5-7 Fr. Out of the 13 valves, 10 were single leaks (76.9\%) and were all closed by a single occluder. There were 2 double leaks (15.3\%) which were closed by two single bigger devices, while the remaining double leak was corrected by two separate devices. The median duration of the procedure was 82 minutes (IQR, $65-85$ minutes). In addition, the mean postoperative ICU stay was 4 days (IQR, 1-2 days) and mean duration from procedure to hospital discharge was 7 days (IQR, 4-7 days). One patient died prior to the discharge.

Conclusion: Taken together, our data demonstrates that percutaneous TA is safe and is associated with a low complication rate and procedure-related mortality. Multiple leaks and diameter of $\geq 10 \mathrm{~mm}$ might increase the risk for residual regurgitation.

\section{Background}

Paravalvular leak (PVL) is a frequent and serious complication after surgical valve replacement or transcatheter valve implantation (1). PVL occurs in 7$17 \%$ of prosthetic valves in the mitral position and $2-10 \%$ in the aortic position $(2,3)$. Patients with PVL present with chronic cardiac insufficiency, hemolytic anemia, or both. Although re-operation is the gold standard for the treatment of PVL, it's usually associated with a high mortality rate (4). More recently, the percutaneous transapical (TA) closure has been adopted as an alternative to the surgical repair and percutaneous transcatheter closure in the treatment of PVL $(5,6)$. However, there is limited data on the utility and safety of the use of percutaneous TA approach for PVLs device closure. Here, we review and demonstrate the efficacy and safety of the percutaneous TA approach for PVL closure in patients with mid-term results.

\section{Methods}

\section{Patients}

This study analyzed the registry of patients who underwent percutaneous closure of the transapical PVL between April 2015 and November 2018 , at our.hospital. All the patients had clinical symptoms or hemolysis and underwent PVL diagnosis by trans-thoracic echocardiography (TTE).. We collected all the clinical reports, examination and imaging data for the analysis. Efficacy endpoints included procedural success and freedom from repeat procedures. On the other hand, safety endpoints included rate of complications of apical access point, pericardial tamponade, pericardial effusion, discharge, 30-day, and 1- or 3-year survival. We defined access-site complication as extravasation from the TA site, major coronary artery compromise, or embolization of a TA site closure device. Additional procedure-specific endpoints for patients undergoing PVL closure included the number and types of devices used or the presence of residual defects.

\section{Preoperative skin-to-apex pathway guiding}

All the patients underwent echocardiography and three dimensional-computerized tomography (3D-CT) evaluation before the procedure. The 3D-CT evaluated the ribs, myocardium, main coronary artery, left ventricular cavity, paravalvular structure and lung tissue, as well as their spatial relationship. The apex puncture point and PVL were used as endpoints and formed a three-dimensional simulation line (Figure 1). Without compromising the papillary muscles, main coronary branches or overlapping lung tissue, the simulation line extended outward through a costal space and to the body surface. An intersection point with the body surface was regarded as the puncture site. The transverse distance between the puncture site and the midline in a CT axial view was measured (Figure $2 \mathrm{~A}$ ). The above virtual space line was projected onto the coronal, sagittal and axial planes.

The virtual axis located on the patients' coronal plane and perpendicular to the patients' long axis was denoted as the $X$ axis. The axis that paralleled the patients' long axis in the same plane was denoted as the $Y$ axis, while the axis perpendicular to the coronal plane was defined as the $Z$ axis. The angle between the virtual line projection on the coronal plane and the $\mathrm{X}$ axis $(\angle 1)$, the angle between the sagittal plane projection and the $\mathrm{Y}$ axis $(\angle 2)$, and the angle between the axial section projection and the $X$ axis $(\angle 3)$ were all measured (Figure $2 B, C$ and $D$ ). Using the above information, we simulated and guided the skin-to-apex puncture.

\section{Percutaneous transapical device closure}

The percutaneous TA closure was conducted under general anesthesia and with cardiopulmonary bypass. The number, size, position, and morphology of PVL was re-evaluated by transesophageal echocardiography (TEE) prior to the procedure. The target intercostal space and the transverse distance were both used to confirm the puncture site. The operation was carried out in the virtual space as determined by the above XYZ axis. 
After systemic heparinization $(1 \mathrm{mg} / \mathrm{kg}$ ), the anesthesiologist temporarily disconnected the ventilator tube, to allow the lung to collapse and then the surgeon undertook the puncture exercise. The $20 \mathrm{G}$ over-the-needle catheter was used, and in cases of blood return, the needle was confirmed by the intraoperative TEE. A guide wire was then introduced into the left ventricle and passed through the PVL under continuous TEE guidance. The introduction of the wire was done in such a manner to avoid any interference to the valve function. The needle was then replaced by a delivery sheath that was advanced through the chest wall into the left ventricle. Depending on the number, size, and morphology of the leak, as well as distance to the prosthetic valve leaflets, a most appropriate occluder was selected and deployed. We gently pulled and pushed on the fixation wire to ascertain a stable position, and if there was a moderate or more residual regurgitation, the deployed occluder was retrieved and replaced by a larger one or multiple occluders (Figure

3). The device was released after confirming its position, as well as the presence or absence of residual shunting and the valvular status. We kept the sheath in the left ventricle and deployed another muscular ventricular septal defect occlude (5-8mm size) at the apical access point. The position of each disc of the second occluder was thoroughly checked before release, to ensure its hemostatic effect. We removed the cable and injected the Surgiflo hemostatic matrix (Ethicon, Somerville, NJ) into the delivery sheath to fill the disc outside the epicardium and tract, from the epicardium to the skin. No additional stitch was needed for the skin puncture site. The absence of pericardial and pleural effusion was checked before leaving the procedure room.

\section{Postoperative management and follow-up}

All the patients in the ICU were closely monitored. The patients received TTE examination on the first postoperative day, a day before discharge, and during the follow-up period. The position and stability of the device, the valvular status, residual regurgitation as well as pericardial effusions were carefully evaluated during the examinations.

\section{Statistical analysis}

The continuous variables were presented as mean \pm standard deviation, while the categorical variables were summarized as numbers and percentage. The statistical analysis was performed using the SPSS statistical package (version 24.0, IBM SPSS, Chicago, Illinois). A $p$ \ 0.05 was considered to be statistical significant.

\section{Result}

Twelve patients (75\% male; median age, 53 years; interquartile range [IQR], 45-60 years,) underwent percutaneous TA approach for PVLs closure during the study period (Table 1). All the 12 patients got 17 prosthetic mechanical valves in mitral and/or aortic position. A total of 13 artificial valves developed PVLs in which 10 of them had a single leak while 3 had double leaks. Characterization of the patients showed that, before the procedure, 8 patients (66.7\%) had atrial fibrillation, while 5 patients $(41.7 \%)$ had trivial to moderate tricuspid regurgitation. Besides, 1 patient had previous coronary artery bypass graft surgery. Four patients had hemolysis before operation, while 3 patients were referred from the cardiology department. The characteristics of these patients are shown in Table 1.

\section{Percutaneous Perventricular Device Closure}

All the TA punctures were conducted successfully. Total median procedure time was 82 minutes (IQR, $65-85$ minutes). The delivery sheath sizes ranged from 5-7 Fr. All the PVL occluders were muscle ventricular septal defect occluder, with a waist height of $5 \mathrm{~mm}$ or $6 \mathrm{~mm}$ (Beijing Starway Medical Technology Inc, Beijing, China). All the apical puncture points were closed with muscle ventricular septal defect occluder with a waist height of $5 \mathrm{~mm}$ or $6 \mathrm{~mm}$ (Beijing Starway Medical Technology Inc, Beijing, China). The size of the PVL occluder ranged from $6 \mathrm{~mm}-12 \mathrm{~mm}$, and the device size for apical access point ranged from $5 \mathrm{~mm}-8 \mathrm{~mm}$. No patient needed sternotomy incision. 10 of the 13 valves $(76.9 \%)$ with a single leak were closed by a single occlude while $2(15.3 \%)$ valves with double leaks were closed by a single bigger device. The last valve with double leaks was corrected by two devices (Table 2).

There was no valve dysfunction, device migration or embolization after deployment of the occluder. 1 patients (10\%) experienced hemodynamically insignificant pericardial effusion after the procedure and there was no new post-operation hemolysis. Compared to pre-procedure, 2 patients had less severe hemolysis while 1 patient had more severe hemolysis. Three patients had residual shunt that led to reoperation in one patient. The patient had significant comorbidity such as viral hepatitis $\mathrm{C}$ and decompensated hepatic cirrhosis, permanent atrial fibrillation and prosthetic valve stenosis in aortic position before surgery. In addition, the patient had two leaks $(8 \mathrm{~mm} / 10 \mathrm{~mm})$ in mitral position with severe regurgitation, NYHA IV and serious anemia. There was mild residual regurgitation after deployment of two devices $(10 \mathrm{~mm} / 12 \mathrm{~mm})$. Our TTE analysis demonstrated an increase in the residual regurgitation, one week after the procedure. The patient had a concurrent secondary serious hemolytic anemia which couldn't be improved by blood transfusion, and an acute renal failure (ARF) which depended on hemodialysis therapy. Successful re-closure to deploy the device decreased the residual regurgitation. However, the patient died due to congestive heart failure, hemolytic anemia, ARF, and multiple organ dysfunction syndrome. The remaining 11 patients were discharged uneventfully.

The mean postoperative ICU stay and mean time from procedure to hospital discharge was 4 days (IQR, 1 - 2 days) and 7 days (IQR, 4 - 7 days) respectively. Only 1 ICU patient stayed for 30 days and died prior to discharge (Table 3).

The median follow-up was 31 (IQR, 16 - 47) months. A total of 11 patients (100\%) and 4 patients (36.3\%) successfully completed the one-year and threeyear follow-up respectively. THE patients took warfarin regularly, and examined international normalized ratio (INR) in order to avoid stroke and bleeding. Our standard TTE analysis showed that one patient had mild residual regurgitation, while another had hemolysis and disappeared at 1 year follow-up. 
Functional status was NYHA class I in six (66.7\%) and II in three (33.3\%) at final follow-up. No patient presented with significant congestive heart failure, cardiovascular events or neurological morbidities (Table 4).

\section{Comment}

Paravalvular leak is defined as peri-prosthetic regurgitation via a defect between sewing ring and the annulus of the native valve (7). Factors such as annular calcification, endocarditis, tissue friability, chronic inflammatory disease, and surgical techniques lead to the occurrence of PVL (8). With the development of medical technology, surgical techniques have become less invasive or even non-invasive. Hourihan $\mathrm{M}$, et al, 1992, first showed the application of TA approach in PVL closure $(6,7,9-11)$.

Compared with the use of femoral vessel, TA approach has excellent technical advantages. TA uses a more direct manipulation of guide-wires and sheaths which results into easier accessibility of defects and a stable implantation of the device. In addition, patients are free from contrast agent examination. Richard Tanner et al. reported a median procedure time of 140 minutes [90-210] (12). Joseph et al. reports were completed successfully via the TA approach with relatively low median fluoroscopy time (26.5 minutes; IQR, 8.3-43.8 minutes) and median procedure time (106 minutes; IQR, 39117 minutes) (7). In our study, we showed that there was no patient who needed screening, and the median procedure time was (81 minutes; IQR, $65-84$ minutes).

Besides, data concerning the safety and utility of percutaneous TA access remains very scant. Here, we demonstrate our experiences with this technique, and review the efficacy and safety of the TA approach to PVL closure. Our analysis only showed a single death $₫ 8.3 \% \bigotimes$ within 30 days. This rate is slightly higher than other larger trials that have reported 30 -day mortality rates of $1.7-4.5 \%$ with percutaneous PVL closure. Surgical series have reported a 30 day mortality rate of $6.9-10.7 \%$ for surgical PVL closure. Percutaneous PVL closure is emerging as the primary strategy for patients with PVL, with surgical PVL closure being reserved for those who fail or have unfavorable anatomy for percutaneous PVL closure.

Our study reported no death during follow-up, and the residual regurgitation and NYHA function class remained stable. Whereas there is limited literature on TA approach to PVL closure, it is reported to be suitable for high risk patients and achieves acceptable periprocedural rate of adverse events. A study by Ruiz et al. reported long-term survival of percutaneous PVL closure at 6,12 and 18 months as $91.9,89.2$ and $86.5 \%$, respectively (5). In addition,

Sorajja et al. showed a 1-2 year survival of 70-75\% after percutaneous PVL closure, with an estimated 3-year survival rate of $64.5 \%$ (13). The direct, inline access to the valve and valve annulus from TA access is therefore attractive in terms of success rate and procedure duration.

To date, there are no specialized devices for transcatheter PVL closure $(14,15)$. We use a VSD occluder in all patients. For different PVLs, occluder waist or height might differ. For the mitral valve leakage, we choose an occluder with waist height of $4 \mathrm{~mm}$ for the CarboMedics mechanical valve and a waist height of $5 \mathrm{~mm}$ for the St. Jude Medical mechanical valve. For the aortic valve leakage, we choose an occluder with waist height of $6 \mathrm{~mm}$ for the CarboMedics supra-annular Top Hat valve and a waist height of $5 \mathrm{~mm}$ for the standard St. Jude mechanical valve. All the puncture site devices have a waist height of $5 \mathrm{~mm}$.

There was residual regurgitation in 3 patients. 2 cases had 2 leaks on the same valve, and the maximal diameter for the larger leak was $\geq 8$ mm, with residual regurgitation. The other patient had a single leak, with a maximal diameter $\geq 10 \mathrm{~mm}$, and a mild or trace residual regurgitation after procedure.

All cases had 2 PVLs of $>=10 \mathrm{~mm}$, and residual shunt as well as hemolysis were found after the closure. Despite PVL reduction to a mild degree in one patient, the hemolysis lasted for 1 year of follow up. We observed that, multiple leaks with diameters of $\geq 10$ mm may be the risk factors for residual defect after PVL closure.

Hemothorax is the most common complication of Percutaneous TA access. Pitta et al. reported occurrence of hemothorax in 6 of 32 (19\%) patients of Percutaneous TA access (16). On the other hand, Zorinas et al. published outcomes of 19 patients who underwent surgical TA catheter-based mitral PVL closure. 1 (5.2\%) patient with hemothorax due to surgical retractor blade injury to the rib with subsequent severe bleeding required surgical revision (17). In our study, 1(8.3\%) patients had hemodynamically insignificant pericardial effusion, after the procedure. Our data demonstrates low procedure-related complications associated with routine closure of TA puncture sites with a device.

In the past decade, there has been a huge application of percutaneous PVL closure. However, the use of the percutaneous PVL closure remains relatively novel, and remains technically challenging especially due to the need for precise size, number and location of PVL and puncture point locations. Cautious evaluation of the coronary artery before "blind" puncture is essential. To ensure the puncture site does not compromise the coronary vasculature, all the patients in our study underwent CTA to precisely define the puncture site and angle before surgery. In addition, the use of CTA is beneficial in cases with unclear anatomy or in patients with poor echocardiography windows. We also routinely ask the anesthesiologist for solitary right-lung ventilation in order to deflate the left lung. Joseph's team (7) innovatively combined CTA with fluoroscopy technology, and then guided by TEE and achieved accurate positioning of the device in hybrid operation room. Compared with this technology, our study only used 3D-CTA imaging before operation, and completed positioning with software post-processing, which does not depend on intraoperative fluoroscopy or hybrid operation room. The whole procedure can performed only under TEE. We therefore observe that hospitals can carry out such operations without hybridized operating rooms.

\section{Conclusion}

The TA approach to PVL closure is a direct approach and significantly reduces the procedure duration. Device closure of the apical access site is reliable and should be adopted. However, there is no literature on the benefits of the TA approach to PVL closure in high risk patients, compare to re-operation.

Page $4 / 10$ 
Appropriate sizing as well as careful intraprocedural guiding are essential in the achievement of this goal. Besides, from our experience, multiple leaks and diameters of $\geq 10 \mathrm{~mm}$ might increase the risk for residual regurgitation. More large scale studies are needed to analyze and ascertain the risk factors.

\section{Abbreviations}

PVL: Paravalvular leaks; TA:percutaneous transapical; TEE:transesophageal echocardiography; 3D:three dimensional; NYHA:New York Heart Association; ARF:acute renal failure; ICU:Intensive Care Unit; VSD:Ventricular septal defect; CTA:Computed Tomography Angiography; CT:Computed Tomography.

\section{Declarations}

\section{Ethics approval and consent to participate}

Biomedical ethics committee of West China Hospital of Sichuan University(Approval number: 2019-547) approved this study. Participants gave written informed consent for analysis and publication.

\section{Consent for publication}

All authors have given final approval of the version to be published.

\section{Availability of data and material}

The authors do not wish to share our data, because the patients' who participated this study did not agree to share their individual data

\section{Competing interests}

The authors declare that they have no competing interests

\section{Funding}

This work was supported in part by the Applied Basic Research Programs of Departmentof Science and Technology of Sichuan Province (20YYJC3415). Funders had no role in study design or interpretation of the findings.

\section{Authors' contributions}

WQR, CPG and KL planned the study. WQR and QY collected the data.

WQR, QY and KL performed the statistical analysis and drafted the manuscript.

All authors read and approved the final manuscript.

\section{Acknowledgements}

Not applicable

\section{References}

1. Yoon S, Whisenant BK, Bleiziffer S, Delgado V, Dhoble A, Schofer N, et al. Outcomes of transcatheter mitral valve replacement for degenerated bioprostheses, failed annuloplasty rings, and mitral annular calcification. EUR HEART J. 2019 2019-02-01;40(5):441-51.

2. Taramasso M, Maisano F, Latib A, Denti P, Guidotti A, Sticchi A, et al. Conventional surgery and transcatheter closure via surgical transapical approach for paravalvular leak repair in high-risk patients: results from a single-centre experience. European Heart Journal - Cardiovascular Imaging 2014 2014-10-01;15(10):1161-7.

3. Bernard S, Yucel E. Paravalvular Leaks-From Diagnosis to Management. Curr Treat Options Cardiovasc Med. 2019 2019-11-14;21(11):67.

4. Azevedo Al, Braga P, Rodrigues A, Santos L, Melica B, Ribeiro J, et al. Percutaneous closure of periprosthetic paravalvular leaks: A viable alternative to surgery? REV PORT CARDIOL. 2017 2017-01-01;36(7-8):489-94.

5. Ruiz CE, Jelnin V, Kronzon I, Dudiy Y, Del Valle-Fernandez R, Einhorn BN, et al. Clinical Outcomes in Patients Undergoing Percutaneous Closure of Periprosthetic Paravalvular Leaks. J AM COLL CARDIOL. 2011;58(21):2210-7.

6. Murakami T, Suehiro Y, Nishimura S, Sugioka K, Iwata S, Ito A, et al. Transcatheter closure through transapical access for mitral paravalvular leak after previous trans-septal mitral operation. General Thoracic and Cardiovascular Surgery. 2017;65(5):289-92.

7. Venturini JM, McClelland I, Blair J, Narang A, Kalathiya R, Lang RM, et al. Percutaneous Transapical Left Ventricular Access to Treat Paravalvular Leak and Ventricular Septal Defect. J INVASIVE CARDIOL. 2019 2019-09-01;31(9):247-52.

8. Binder RK, Webb JG. Percutaneous Mitral and Aortic Paravalvular Leak Repair: Indications, Current Application, and Future Directions. CURR CARDIOL REP. 2013;15(3). 
9. Hourihan M, Perry SB, Mandell VS, Keane JF, Rome JJ, Bittl JA, et al. Transcatheter umbrella closure of valvular and paravalvular leaks. J AM COLL CARDIOL. 1992 1992-01-01;20(6):1371-7.

10. Jelnin V, Dudiy Y, Einhorn BN, Kronzon I, Cohen HA, Ruiz CE. Clinical experience with percutaneous left ventricular transapical access for interventions in structural heart defects a safe access and secure exit. JACC Cardiovasc Interv. 2011 2011-08-01;4(8):868-74.

11. Jelnin V, Dudiy Y, Einhorn BN, Kronzon I, Cohen HA, Ruiz CE. Clinical Experience With Percutaneous Left Ventricular Transapical Access for Interventions in Structural Heart Defects. JACC: Cardiovascular Interventions. 2011;4(8):868-74.

12. Tanner R, Hassan S, Ryan N, Murphy NF, Campbell P, Margey R, et al. Trans-catheter paravalvular leak closure: a single-centre experience. Irish Journal of Medical Science (1971 -). 2019;188(2):489-96.

13. Sorajja P, Cabalka AK, Hagler DJ, Rihal CS. Long-term follow-up of percutaneous repair of paravalvular prosthetic regurgitation. J AM COLL CARDIOL. 2011 2011-11-15;58(21):2218-24.

14. Okutucu S, Mach M, Oto A. Mitral paravalvular leak closure: Transcatheter and surgical solutions. Cardiovascular Revascularization Medicine. 2019.

15. Gafoor S, Franke J, Bertog S, Lam S, Vaskelyte L, Hofmann I, et al. A Quick Guide to Paravalvular Leak Closure. Interv Cardiol. 2015 2015-0501;10(2):112-7.

16. Pitta SR, Cabalka AK, Rihal CS. Complications associated with left ventricular puncture. CATHETER CARDIO INTE. 2010 2010-12-01;76(7):993-7.

17. Zorinas A, Janusauskas V, Davidavicius G, Simakauskas R, Puodziukaite L, Zakarkaite D, et al. Retrospective analysis of single-center early and midterm results of transapical catheter-based mitral paravalvular leak closure with a purpose-specific device. Postepy Kardiol Interwencyjnej. 2018 2018-01-20;14(2):167-75.

\section{Tables}

Table 1. Baseline and procedural characteristics.

\begin{tabular}{|ll|}
\hline Variables & $(\mathrm{n}=12)$ \\
\hline Age,years & 53 [IQR,7] \\
\hline Male & $75 \%$ \\
\hline Hemolytic anemia & $4(33.3 \%)$ \\
\hline NYHA functional class & \\
\hline Class II & $4(33.3 \%)$ \\
\hline Class III & $7(58.3 \%)$ \\
\hline Class IV & $1(8.3 \%)$ \\
\hline Permanent atrial fibrillation & $8(66.7 \%)$ \\
\hline Tricuspid insufficientcy & $5(41.7 \%)$ \\
\hline Coronary artery disease & $1(8.3 \%)$ \\
\hline Prosthetic valve stenosis & $1(8.3 \%)$ \\
\hline Diabete & $1(8.3 \%)$ \\
\hline Hepatic failure & $1(8.3 \%)$ \\
\hline Transferred from cardiology department & $3(25 \%)$ \\
\hline
\end{tabular}

Data provided as number(\%) or median(interquartile range[IQR])

NYHA, New York Heart Association.

\section{Table 2. Patients and procedures}




\begin{tabular}{|c|c|c|c|c|c|c|c|c|c|}
\hline patient & $\begin{array}{l}\text { PVL } \\
\text { Location }\end{array}$ & $\begin{array}{l}\text { PVL } \\
\text { Numbers }\end{array}$ & PVL Diameter & $\begin{array}{l}\text { Regurgitational } \\
\text { degree }\end{array}$ & $\begin{array}{l}\text { Hemolytic } \\
\text { anemia(N/Y) }\end{array}$ & $\begin{array}{l}\text { sheath } \\
\text { sizes }\end{array}$ & Device size & $\begin{array}{l}\text { Residual } \\
\text { regurgitation }\end{array}$ & $\begin{array}{l}\text { Hemolytic } \\
\text { anemia } \\
\text { postoperation }\end{array}$ \\
\hline$\# 1$ & MV & 1 & $8 * 5 \mathrm{~mm}$ & III & $\mathrm{N}$ & $7 F$ & $10 \mathrm{~mm}$ & 0 & $\mathrm{~N}$ \\
\hline \#2 & MV & 1 & $5 \star 3 \mathrm{~mm}$ & III & $\mathrm{Y}$ & $5 F$ & $6 \mathrm{~mm}$ & 0 & $\mathrm{~N}$ \\
\hline \#3 & AV & 1 & $11 * 9 \mathrm{~mm}$ & III & $\mathrm{Y}$ & $7 F$ & $12 \mathrm{~mm}$ & 1 & $\mathrm{Y}$ \\
\hline$\# 4$ & MV & 1 & $5 \star 5 \mathrm{~mm}$ & II & $\mathrm{N}$ & $6 \mathrm{~F}$ & $7 \mathrm{~mm}$ & 0 & $\mathrm{~N}$ \\
\hline \#5 & MV & 2 & $8 * 7 \mathrm{~mm} / 4 * 4 \mathrm{~mm}$ & III & $\mathrm{N}$ & $7 F$ & $10 \mathrm{~mm}$ & I & $\mathrm{N}$ \\
\hline$\# 6$ & MV & 1 & $6 * 3 \mathrm{~mm}$ & II & $\mathrm{N}$ & $5 F$ & $6 \mathrm{~mm}$ & 0 & $\mathrm{~N}$ \\
\hline \#7 & MV & 1 & $10 * 4 \mathrm{~mm}$ & II & $\mathrm{N}$ & $7 F$ & $12 \mathrm{~mm}$ & 0 & $\mathrm{~N}$ \\
\hline \multirow[t]{2}{*}{$\# 8$} & MV & 1 & $6 * 2 \mathrm{~mm}$ & II & $\mathrm{N}$ & $7 F$ & $7 \mathrm{~mm}$ & 0 & $\mathrm{~N}$ \\
\hline & $\mathrm{AV}$ & 2 & $8 * 4 \mathrm{~mm} / 6 * 3 \mathrm{~mm}$ & III & & & $12 \mathrm{~mm}$ & 0 & \\
\hline \#9 & MV & 2 & $10 * 6 \mathrm{~mm} / 8 * 6 \mathrm{~mm}$ & III & $\mathrm{Y}$ & $7 F$ & $12 \mathrm{~mm} / 10 \mathrm{~mm}$ & II & $\mathrm{Y}$ \\
\hline \#10 & MV & 1 & $6 * 4 \mathrm{~mm}$ & II & $\mathrm{N}$ & $6 \mathrm{~F}$ & $7 \mathrm{~mm}$ & 0 & $\mathrm{~N}$ \\
\hline \#11 & AV & 1 & $8 * 5 \mathrm{~mm}$ & II & $\mathrm{N}$ & $6 \mathrm{~F}$ & $10 \mathrm{~mm}$ & 0 & $\mathrm{~N}$ \\
\hline \#12 & MV & 1 & $8 * 4 \mathrm{~mm}$ & II & $Y$ & $6 \mathrm{~F}$ & $10 \mathrm{~mm}$ & 0 & $\mathrm{~N}$ \\
\hline
\end{tabular}

MV:mitral valve, AV:aortic valve, Y:yes, N:no, Device size: Ventricular septal occluder diameter, PVL is classified according to the Valve Academic Research Consortium (VARC)-2 Consensus as: 0 (none), I (mild), II (moderate), or III (severe)

Table 3. Procedural outcomes

\begin{tabular}{|ll|}
\hline Variables & \\
\hline Residual regurgitation & \\
\hline 0 & 9 \\
\hline I & 2 \\
\hline II & 1 \\
\hline III & 0 \\
\hline Technical success & 12 \\
\hline Complication & \\
\hline Hemolytic anemia & 2 \\
\hline Valve dysfunction & 0 \\
\hline Device migration & 0 \\
\hline Embolization & 0 \\
\hline Pericardial effusion & 1 \\
\hline Re-operation & 1 \\
\hline Hospital deaths & 1 \\
\hline Median ICU stays & 4 days (IQR, 1-2 days) \\
\hline Median Hospital stays & 7 days (IQR, 4-7days) \\
\hline
\end{tabular}

Residual regurgitation is classified according to the Valve Academic Research Consortium (VARC)-2 Consensus as: 0 (none), I (mild), II (moderate), or III (severe) 


\begin{tabular}{|ll|}
\hline Variables & \\
\hline Number of follow-up & 11 \\
\hline Follow-up time, months & 31 (IQR, 16-47) \\
\hline Hemolytic anemia & 0 \\
\hline NYHA functional class & \\
\hline Class I & 5 \\
\hline Class II & 2 \\
\hline Class III & 0 \\
\hline Class IV & 0 \\
\hline Residual regurgitation & 1 \\
\hline Death & 0 \\
\hline Re-operation & 0 \\
\hline
\end{tabular}

Data are presented as the number of patients (percentage) or mean \pm standard deviation. NYHA, New York Heart Association.

\section{Figures}
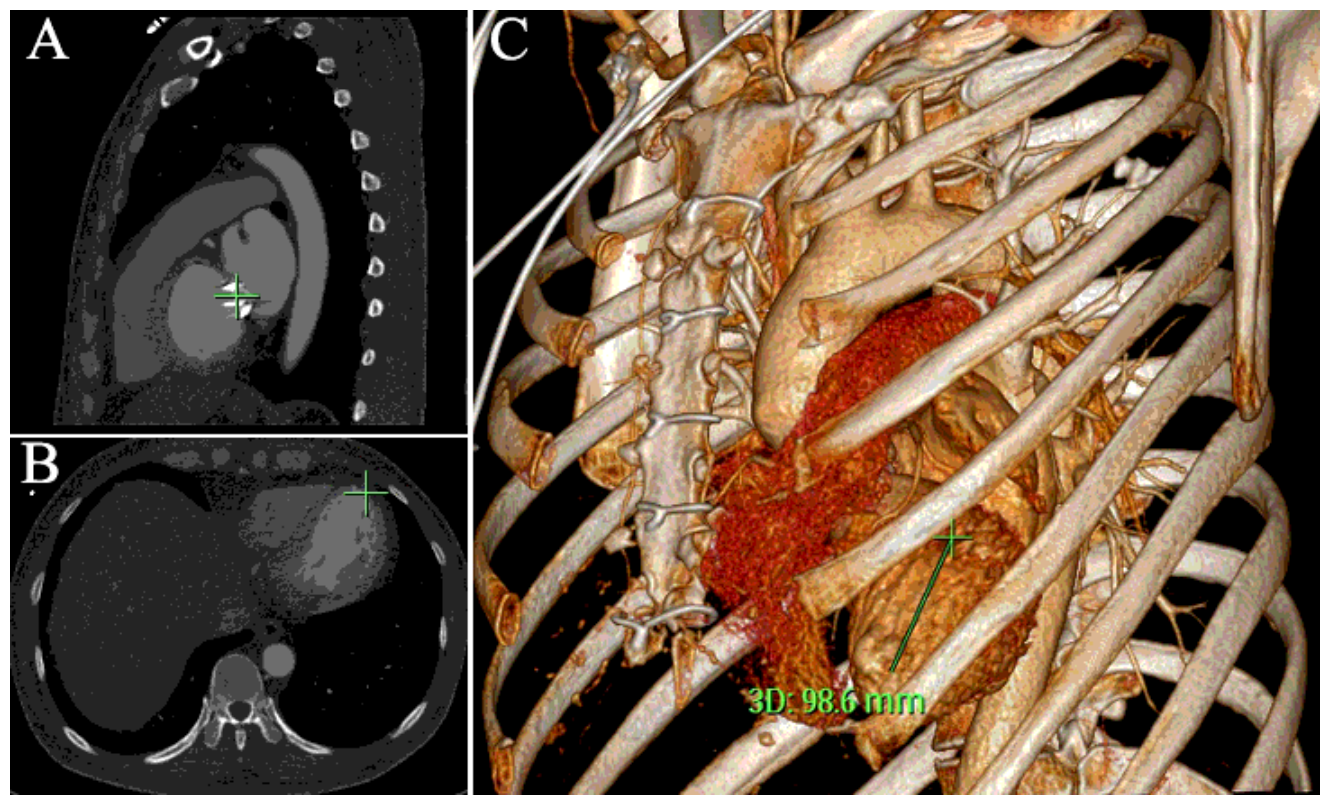

Figure 1

Three dimensional-computer tomography showed perivalvular leakage(A), apical puncture point $(B)$, and simulation puncture line(C) 

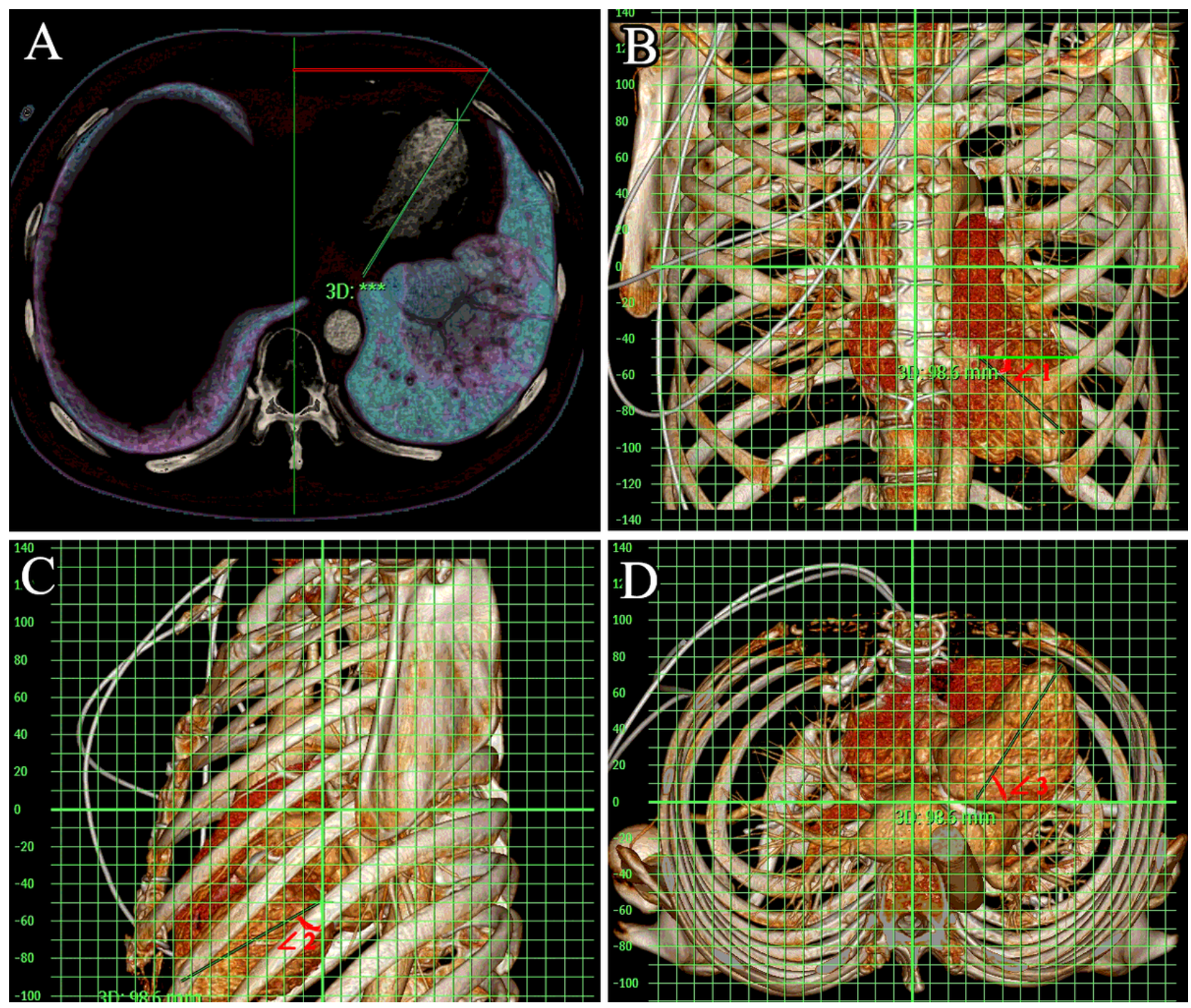

Figure 2

Three dimensional-computer tomography showed the vertical distance between the body surface puncture point and the midline(A, red line), and the angle between the line projection of the coronal plane and the $\mathrm{X}$ axis $(\angle 1, \mathrm{~B})$, the angle between the sagittal plane projection and the $\mathrm{Y}$ axis $(\angle 2, \mathrm{C})$, and the angle between the cross section projection and the $\mathrm{X}$ axis $(\angle 3, \mathrm{D})$.

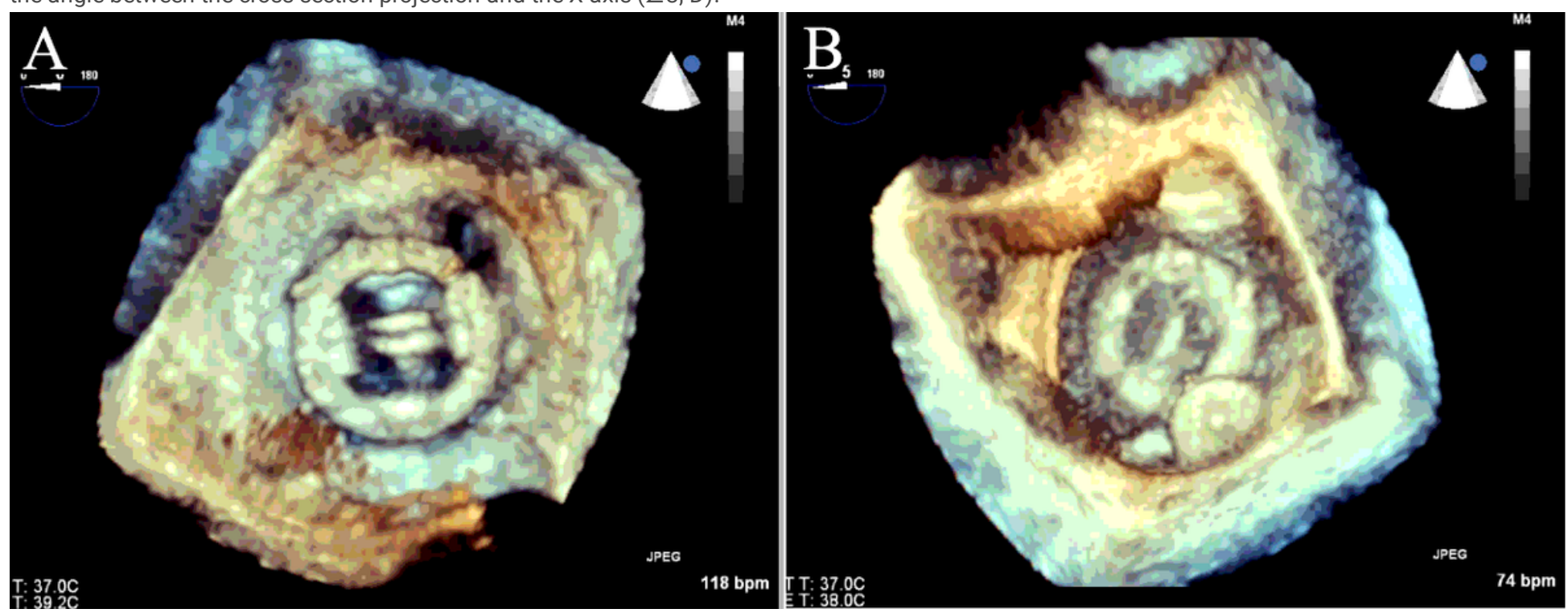

Figure 3 
Three dimensional transesophageal echocardiography (3D-TEE) suggested the PVL at pre-closure(A) and occluder at post-closure(B).

Page $10 / 10$ 